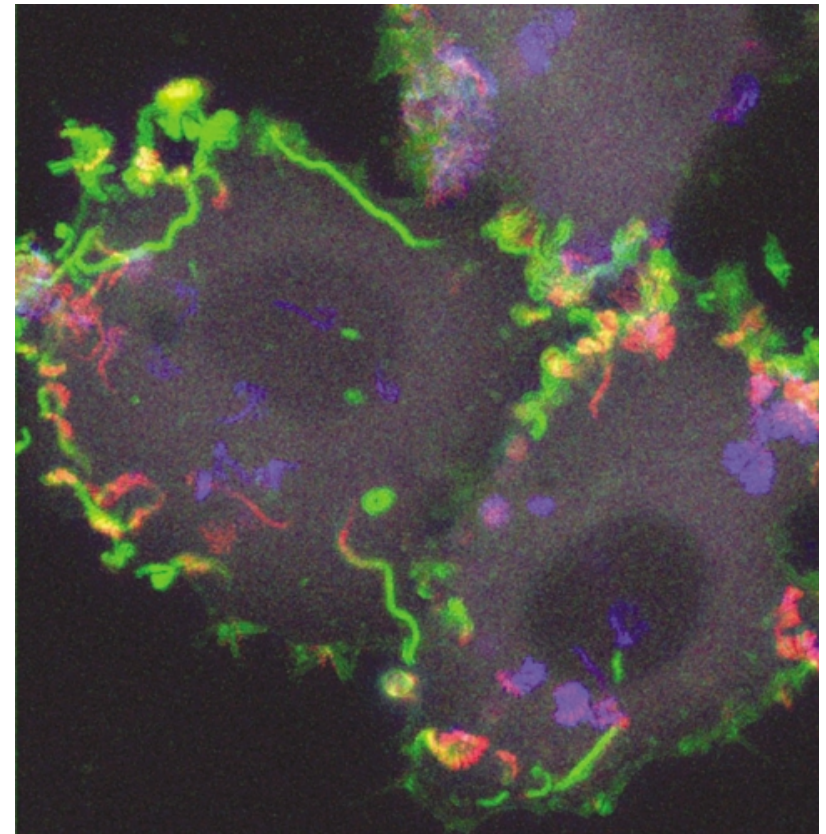

CELLULAR MICROBIOLOGY

\section{Vaccinia virus on the move}

Vaccinia virus is closely related to the virus that causes smallpox, and was used as a vaccine to eradicate this disease. Vaccinia replicates in the cytoplasm, then moves to the cell surface from where it spreads to neighbouring cells. Virus particles have been shown to induce the formation of actin tails, on which they are thought to be propelled through the cytoplasm to reach the plasma membrane. After fusion with the membrane, virus particles are found on the tips of cellular protrusions that are driven by actin polymerization. Three recent papers now modify our view on how vaccinia moves - and implicate a second cytoskeletal transport system.

The new reports show that actin-based motility only occurs close to the cell periphery, and that the formation of actin tails seems to take place only during or after fusion of viral particles with the plasma membrane. To reach the cell periphery, however, vaccinia particles travel along microtubules in a saltatory fashion. This is the first example of a virus using both the microtubule and actin cytoskeleton to spread from cell to cell.

Rietdorf and colleagues have identified conventional kinesin as the motor that is responsible for microtubule-based transport, and show that this motor is recruited to viral particles through the tetratricopeptide (TPR) repeats in the kinesin light chain. The A36R protein, which is encoded by vaccinia and is essential for vaccinia-induced actin polymerization, is also required to recruit kinesin to virus particles and for their movement to the cell periphery. Interestingly, Rietdorf et al. found that virus particles are never associated with both kinesin and actin tails, indicating that the transition from movement along microtubules to fusion with the plasma membrane and actin-tail formation is tightly regulated. Whether A36R has any role in this, and the nature of the exact molecular link between A36R and kinesin-dependent movement, will be the subject of future studies.

Barbara Marte, Editor, Nature Cell Biology

\section{(i) References and links}

ORIGINAL RESEARCH PAPERS Ward, B. M. \& Moss, B. Visualization of intracellular movement of vaccinia virus virions containing a green fluorescent protein-B5R membrane protein chimera. J. Virol. 75, 4802-4813 (2001) | Hollinshead, M. et al. Vaccinia virus ulitilizes microtubules for movement to the cell surface. J. Cell Biol. 154, 389-402 (2001) | Rietdorf, J. et al. Kinesin-dependent movement on microtubules precedes actin-based motility of vaccinia virus. Nature Cell Biol. 3, 992-1001 (2001)

\title{
TELOMERES
}

\section{Short, but not sweet}

Telomere shortening can lead to genomic instability and cancer, as telomeres protect the chromosome ends from being recognized as DNA breaks, which prevents chromosome end-to-end fusions. So what determines the cellular response to telomere shortening? Several groups have proposed that it is the average telomere length, but now, Carol Greider's group, reporting in Cell, show that it is the shortest telomere that initiates the response and affects cell survival.

To investigate this problem, the authors crossed mice that had long telomeres (heterozygous for telomerase; $m T^{+/-}$) with mice that had short telomeres - deleted for telomerase and interbred for six generations $\left(m T R^{-1} \mathrm{G} 6\right)$. The offspring would have a similar average telomere length, which would be equivalent to $m T R^{-1-}$ G3 mice, and would be either null (F1 $\left.m T R^{--}\right)$or heterozygous $\left(\mathrm{F} 1 m T R^{+-}\right)$ for telomerase activity. Late-generation mice (G4-G6) show fertility defects, but the $m T R^{--} \mathrm{G} 3$ mice do not. Would the $\mathrm{F} 1 m T R^{-/}$and $\mathrm{F} 1 m \mathrm{mR}^{+/-}$mice? The F1 $m T R^{+/-}$mice have a similar phenotype to the parental $m T R^{+/-}$ strain, but the $\mathrm{F} 1 \mathrm{mTR}^{-/-}$mice are more like the $m T^{-/} \mathrm{G} 6$ mice than the $m T^{-/-} \mathrm{G} 3$ mice. Both strains have a correspondingly low testes weight, which is a reflection of the increased germ-cell apoptosis. The number of chromosome fusions is also higher than in the $m T^{-/-} \mathrm{G} 3$ mice. These results indicate that it is the presence of short telomeres, rather than the average telomere length, that elicits the cellular response.

Interestingly, when the telomerelength distribution was measured in these mice - using quantitative fluorescence in situ hybridization (QFISH) - the authors found that the average telomere length was only slightly higher in $\mathrm{F} 1 \mathrm{mTR}^{+/-}$mice than in $\mathrm{F} 1 \mathrm{mTR}^{-/-}$mice. There was, however, a significant difference in the number of short telomeres in the two mouse strains. It seems that the short telomeres in the $\mathrm{F} 1 \mathrm{mTR}^{+/-}$mice can be specifically recognized and elongated to restore telomere function.
To further characterize the cellular response to telomere shortening, the authors measured chromosome fusions and the number of short telomeres in $m \mathrm{TR}^{-/-}$mice. The fusion events were non-random - for example, fusions frequently involved chromosomes 5 and 19 in one mouse strain - and correlated with chromosomes that had short telomeres. Chromsome fusions are non-random in other $\mathrm{mTR}^{-/-}$strains, but do not involve the same chromosomes. The participation in fusion events is therefore not chromosome-specific. The authors concluded that a short telomere is most likely to lose function and fuse with another short telomere, and therefore that two short telomeres might be required for a fusion event to take place.

So what causes telomere dysfunction and chromosome fusion? Sequencing of the junctions between the two chromosomes showed that no telomere sequence is present; and FISH showed that microsatellite repeats are also decreased. However, regions of microhomology do exist within the remaining microsatellite repeats, which supports the previous hypothesis that non-homologous end joining is involved in chromosome fusions.

One problem that remains to be solved is how telomerase activity is targeted to the shortest telomeres. We await, with interest, further explanation of this mechanism.

Emma Greenwood Associate Editor, Nature Reviews Cancer

\section{(2) References and links} ORIGINAL RESEARCH PAPER Hemann, M. T. et al. The shortest telomere, not average telomere length, is critical for cell viability and chromosome stability. Cell 107, 67-77 (2001) WEB SITE Carol Greider's lab: http://telomerase.bs.jhmi.edu/GreiderLab/index.htm

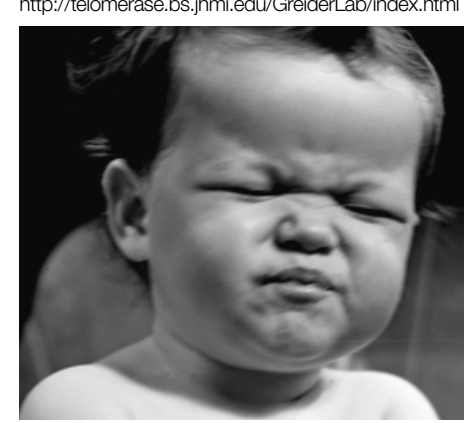

